\title{
A splitting technique for analytical modelling of two-phase multicomponent flow in porous media
}

\section{Introduction}

Enhanced Oil Recovery (EOR) methods include injection of different fluids into reservoirs to improve oil displacement. Displacement of oil by any of these

\footnotetext{
* Corresponding author. Fax: +55 2227969734.

E-mail address: puime@lenep.uenf.br (A.P. Pires).
}

fluids involves complex physico-chemical interphase mass transfer, phase transitions and transport property changes. These processes can be divided into two main categories: that of thermodynamics and of hydrodynamics. They occur simultaneously during the displacement, and are coupled in the modern mathematical models of EOR.

The mathematical models for two-phase Enhanced Oil Recovery processes consist of mass conservation 
for each component closed by thermodynamic relationships of phase equilibria. Thermal EOR models contain also the energy conservation law. The resulting systems of conservation laws (Gelfand, 1959; Dafermos, 2000) are hyperbolic (Logan, 1994). Solutions consist of continuous simple (rarefaction) waves and stable admissible shocks (Kulikovskii and Sveshnikova, 1995; Kulikovskii et al., 2001).

Continuous injection of EOR fluid corresponds to self-similar Riemann problem for the system of twophase multi component flow equations. Injection of EOR fluid slugs with a water/gas drive results in nonself-similar problems of hyperbolic wave interactions.

Exact analytical solutions have been obtained for continuous chemical flooding by one component (Fayers, 1962; de Nevers, 1964; Claridge and Bondor, 1974; Helfferich, 1980), by two components (Braginskaya and Entov, 1980) and by any arbitrary number of components (Johansen and Winther, 1989; Johansen et al., 1989; Dahl et al., 1992). A graphical technique to solve the $(2) \times(2)$ system for two-phase three-component gas flooding was developed and several exact solutions for Riemann problems of continuous gas injection were obtained by Wachman (1964). Other solutions for different types of phase diagrams and boundary conditions related to injection of other fluids were found using the same technique (Hirasaki, 1981; Dumore et al., 1984; Lake, 1989).

Semi-analytical solutions for $n$-component gas flooding were obtained by numerical combination of shocks and rarefactions (Johns et al., 1993; Johns and Orr, 1996; Orr et al., 1995). The reduction of the continuous gas flood system dimension was developed through the lifting of the concentration waves from the system with lower dimension, and the exact solutions were obtained for the displacement of $n$ component ideal mixtures (Bedrikovetsky and Chumak, 1992a,b). These reduction technique and solutions were used for different initial-boundary data corresponding to different gas floods (Entov and Voskov, 2000; Entov et al., 2002). Non-self-similar analytical models for displacement of oil by chemical and gas/solvent slugs were derived explicitly by Bedrikovetsky (1993). The detailed study of these analytical EOR models can be find in monographs by Lake (1989), Barenblatt et al. (1991) and Bedrikovetsky (1993).

It was observed from semi-analytical and numerical experiments on the continuous displacement of oil by gases that several thermodynamic features (MMP, key tie lines, etc.) are independent of transport properties (Zick, 1986; Bedrikovetsky and Chumak, 1992a,b; Orr et al., 1995; Wang and Orr, 1997). The analytical modelling of multicomponent polymer/surfactant flood also allows observing that the concentration "path" of the solution is completely defined by adsorption isotherms and does not depend on relative permeability and phase viscosities (Johansen and Winther, 1989; Johansen et al., 1989; Bedrikovetsky, 1993). Nevertheless, the independence of thermodynamics and hydrodynamics for two-phase multi component flows in porous media has never been proved.

The model for one-dimensional displacement of oil by different EOR fluids is analysed in this paper. The main result is the splitting of thermodynamical and hydrodynamical parts in the EOR mathematical model. The introduction of a potential associated with one of the conservation laws and its use as an independent variable reduces the number of equations by one. The algorithm to solve the problem includes solution of the reduced auxiliary problem, solution of one lifting hyperbolic equation and inversion of the coordinate transformation.

The reduced auxiliary system contains just thermodynamical (equilibrium fractions of each phase, sorption isotherms) variables and the lifting equation contains just hydrodynamical (phases relative permeabilities and viscosities) parameters while the initial EOR model contains both thermodynamical and hydrodynamical functions. So, the problem of EOR displacement was divided into two independent problems: that of thermodynamics and that of hydrodynamics. The number of auxiliary equations is less than the number of equations in the compositional model by one. Explicit projection and lifting procedures are derived. The splitting is valid for either self-similar continuous injection problems or for non-self-similar slug injection problems.

Therefore, phase transitions occurring during displacement are determined by the auxiliary system, i.e. they are independent of hydrodynamic properties of fluids and rock. For example, the minimum miscibility pressure (MMP) and tie line sequences in displacement zones are independent of relative permeabilities and phases viscosities. Relative motion of polymer, surfactant and fresh water/brine slugs depends on sorption isotherms only. The splitting technique was used for the development of analytical model for nonself-similar displacement of oil by polymer slug with water drive.

Presently the development of 1D analytical models becomes particularly important in 3D streamline simulation. With respect to $3 \mathrm{D}$ flows, the splitting takes place only for the case of constant total mobility 
147 (where the stream line concept is valid). For the general 148 case of the total mobility variation, mixing between 149 fluids that enter different streamlines occurs, and split150 ting does not happen any more.

151 In Section 2 we present the splitting method for 152 different two-phase multicomponent flows in porous 153 media that correspond to various EOR methods: chem154 ical flooding is given in 2.1, gasflooding is presented 155 in 2.2, WAG injection is derived in 2.3, carbonised 156 water flooding in 2.4 and non-isothermal waterflood157 ing is presented in 2.5. The analytical model for 1D 158 displacement of oil by a polymer slug with water drive 159 as an illustration of the technique developed is pre160 sented in Section 3. Brief description of various appli161 cations in streamline simulation and laboratory EOR 162 is shown in Section 4. Summary and conclusions are 163 presented in Section 5. Proofs of splitting can be found 164 in Appendixes.

\section{2. Mathematical models of enhanced oil recovery 166 processes}

167 In this part, several systems of equations that arise in 168 enhanced oil recovery processes are presented, and the 169 splitting technique applied.

\section{2.1. Chemical flooding}

171 We consider the linear displacement of oil by an 172 aqueous solution of $n$-components (polymer, salts) in 173 a reservoir of constant permeability and porosity. 174 The reservoir is initially saturated with oil and 175 water. The fluid system contains two incompressible 176 phases (oil and water). There are also n low concen177 tration components dissolved in the aqueous phase, so 178 the change of concentrations does not affect the aque179 ous phase density. The components can be adsorbed 180 by the porous rock. The following conditions are 181 assumed:

182

183 - Neglected capillary pressure and diffusion;

184 - Instantaneous thermodynamics equilibrium;

185 - Constant pressure and temperature.

186

187 Under the conditions of thermodynamic equilibrium, 188 the concentrations of the components adsorbed $\left(a_{i}\right)$ and 189 dissolved in water $\left(c_{i}\right)$ are governed by adsorption 190 isotherms:

$$
\begin{aligned}
& \vec{a}=\vec{a}(\vec{c}), \quad \vec{a}=\left(a_{1}, a_{2}, \ldots, a_{n}\right), \\
& \vec{c}=\left(c_{1}, c_{2}, \ldots, c_{n}\right)
\end{aligned}
$$

The closed system of governing equations includes the conservation laws for the aqueous phase volume and for the mass of each component under equilibrium sorption conditions. The unknowns in the $(n+1) \times(n+1)$ system are the scalar water saturation function $s\left(x_{\mathrm{D}}, t_{\mathrm{D}}\right)$ and the vector-valued function $\vec{c}\left(x_{\mathrm{D}}, t_{\mathrm{D}}\right)$ :

$$
\begin{aligned}
& \frac{\partial s}{\partial t_{\mathrm{D}}}+\frac{\partial f(s, \vec{c})}{\partial x_{\mathrm{D}}}=0 \\
& \frac{\partial(\vec{c} s+\vec{a}(\vec{c}))}{\partial t_{\mathrm{D}}}+\frac{\partial \vec{c} f(s, \vec{c})}{\partial x_{\mathrm{D}}}=0
\end{aligned}
$$

where the following dimensionless coordinates are used:

$x_{\mathrm{D}}=\frac{x}{l}, \quad t_{\mathrm{D}}=\frac{u t}{\Phi l}$

where $\Phi$ is porosity.

The fractional flow function is defined as:

$$
f=f(s, \vec{c})=\left(1+\frac{k_{\mathrm{ro}}(s, \vec{c}) \mu_{\mathrm{w}}}{\mu_{\mathrm{o}} k_{\mathrm{rw}}(s, \vec{c})}\right)^{-1}
$$

Initial and boundary conditions for continuous polymer injection correspond to:

$\left\{\begin{array}{l}s\left(x_{\mathrm{D}}, 0\right)=s^{\mathrm{I}} \\ \vec{c}\left(x_{\mathrm{D}}, 0\right)=0\end{array}\right.$

$\left\{\begin{array}{l}s\left(0, t_{\mathrm{D}}\right)=s^{\mathrm{J}} \\ \vec{c}\left(0, t_{\mathrm{D}}\right)=\vec{c}^{\mathrm{J}}\end{array}\right.$

The boundary conditions for the displacement of oil by a polymer slug with water drive are:

$\vec{c}\left(0, t_{\mathrm{D}}\right): \begin{cases}\vec{c}^{\mathrm{J}}, & t_{\mathrm{D}}<1 \\ 0, & t_{\mathrm{D}}>1\end{cases}$

The conservation law for the aqueous phase allows the introduction of the following potential:

$s=-\frac{\partial \varphi}{\partial x_{\mathrm{D}}}, \quad f=\frac{\partial \varphi}{\partial t_{\mathrm{D}}}$

Consider any trajectory $x_{\mathrm{D}}=x_{\mathrm{D}}\left(t_{\mathrm{D}}\right)$ that starts at $x_{\mathrm{D}}=0$ at the moment $t_{\mathrm{D}}$. The potential $\varphi\left(x_{\mathrm{D}}, t_{\mathrm{D}}\right)$ is the water volume flowing through the trajectory during the period $t_{\mathrm{D}}$ :

$\varphi\left(x_{\mathrm{D}}, t_{\mathrm{D}}\right)=\int_{0,0}^{x_{\mathrm{D}}, t_{\mathrm{D}}} f d t_{\mathrm{D}}-s d x_{\mathrm{D}}$

and the integral (8) is a function of $x_{\mathrm{D}}$ and $t_{\mathrm{D}}$, which is independent of the trajectory. 
221 After the following transformation of independent 222 variables:

$\Theta:\left(x_{\mathrm{D}}, t_{\mathrm{D}}\right) \rightarrow\left(x_{\mathrm{D}}, \varphi\right)$

223 system (2) becomes

$\frac{\partial}{\partial \varphi}\left(\frac{s}{f}\right)-\frac{\partial}{\partial x_{\mathrm{D}}}\left(\frac{1}{f}\right)=0$

$\frac{\partial \vec{a}(\vec{c})}{\partial \varphi}+\frac{\partial \vec{c}}{\partial x_{\mathrm{D}}}=0$

225

228 Derivation of system (11) is presented in Appendix

229 A. The most important feature of the system (10), (11)

230 is the independence of the $n$ equations (11) from the

231 first Eq. (10). The unknowns in the system (11) are $c_{i}$,

$232 i=1,2,3, \ldots, n$. The hyperbolic Eq. (10) contains the

233 unknown $s\left(x_{\mathrm{D}}, \varphi\right)$ and the known vector function

$234 \vec{c}\left(x_{\mathrm{D}}, \varphi\right)$, which is the solution of (11).

235 The system (11) is called the auxiliary system of the

236 large system (2). It is important to mention that the

237 system (2) contains thermodynamic functions and

238 transport properties, while the auxiliary system contains

239 only thermodynamic functions.

240 The initial and boundary conditions (5) and (6) allow 241 the calculation of the potential $\varphi$ where these condi242 tions are set. Integration of the potential Eq. (8), ac243 counting for (5), determines the initial and boundary 244 conditions for continuous chemical injection in plane $245\left(x_{\mathrm{D}}, \varphi\right)$ :

$t_{\mathrm{D}}=0: \varphi=-s^{\mathrm{I}} x_{\mathrm{D}}$

$x_{\mathrm{D}}=0: \varphi=f^{\mathrm{J}} t_{\mathrm{D}}$

248 Then, the initial-boundary conditions (5) become

$$
\begin{aligned}
& \varphi=-s^{\mathrm{I}} x_{\mathrm{D}}\left\{\begin{array}{l}
s=s^{\mathrm{I}} \\
\vec{c}=0
\end{array}\right. \\
& \varphi=-f^{\mathrm{J}} t_{\mathrm{D}}\left\{\begin{array}{l}
s=s^{\mathrm{J}} \\
\vec{c}=\vec{c}^{\mathrm{J}}
\end{array}\right.
\end{aligned}
$$

250 Finally, the boundary conditions (6), for the dis252 placement of oil by a polymer slug with water drive 253 take the form:

$$
\varphi=f^{\mathrm{J}} t_{\mathrm{D}}\left\{\begin{array}{l}
s=s^{\mathrm{J}}, \forall t_{\mathrm{D}} \\
\vec{c}=\vec{c}^{\mathrm{J}}, t_{\mathrm{D}}<1 \\
\vec{c}=0, t_{\mathrm{D}}>1
\end{array}\right.
$$

256 It is possible to prove that any Cauchy or initial257 boundary value problem for the model (2) can be projected onto the corresponding Cauchy or initialboundary value problem for the auxiliary system.

Consider the trajectory $x_{\mathrm{D}}=x_{\mathrm{D}}\left(t_{\mathrm{D}}\right)$ and its image $\varphi=\varphi\left(t_{\mathrm{D}}\right)$ by the mapping $(9)$ :

260

261

$\varphi\left(t_{\mathrm{D}}\right)=\varphi\left(x_{\mathrm{D}}\left(t_{\mathrm{D}}\right), t_{\mathrm{D}}\right)$

Define the trajectory speeds

262

$\begin{aligned} D & =\frac{d x_{\mathrm{D}}}{d t_{\mathrm{D}}} \\ V & =\frac{d x_{\mathrm{D}}}{d \varphi}\end{aligned}$

Using $x_{\mathrm{D}}$ as a parameter for both curves $x_{\mathrm{D}}=x\left(t_{\mathrm{D}}\right)$ and $\varphi=\varphi\left(t_{\mathrm{D}}\right)$ it is possible to obtain

$\frac{1}{V}=\frac{f}{D}-s$

from which follows the relationship between elementary wave speeds in planes $\left(x_{\mathrm{D}}, t_{\mathrm{D}}\right)$ and $\left(x_{\mathrm{D}}, \varphi\right)$ :

260

$D=\frac{f}{s+1 / V}$

For example, the eigenvalues of the large and auxiliary systems for $c$ waves are related by:

272

$\Lambda_{i+1}(s, \vec{c})=\frac{f}{s+1 / \lambda_{i}}, i=1, \ldots, n$.

\subsection{Gas flooding}

Consider 1D two-phase multicomponent gas flooding under the following assumptions:

279

280

281

- Neglected capillary pressure and diffusion;

282

283

- Instantaneous thermodynamic equilibrium;

- Constant pressure and temperature;

284

- Equal component individual densities in both phases.

285

286

287

Thermodynamic equilibrium implies $n-2$ independent phase fractions. We choose components $i=2,3$, $\ldots, n-1$ in gas phase for the vector of independent phase fractions:

288

289

290

291

$\vec{g}=\left(c_{2 g}, c_{3 g}, \ldots, c_{(n-1) g}\right)$

Under the above mentioned conditions, the total two-phase flux is conserved, and $n$ mass balances for $n$-components are replaced by $n-1$ volume conserva- 
297 tion laws for $n-1$ components:

$\frac{\partial C_{i}}{\partial t_{\mathrm{D}}}+\frac{\partial F_{i}}{\partial x_{\mathrm{D}}}=0$

$x_{\mathrm{D}}=\frac{x}{l}, t_{\mathrm{D}}=\frac{u t}{\Phi l}$

298 where the overall $i$-th component fraction and flux are

$C_{i}-c_{i l} S+c_{i \mathrm{~g}}(1-S)$

$300 F_{i}=c_{i l} f+c_{i g}(1-f)$

$30 B$ Here $f$ is the fractional flow of liquid:

$f(S, \vec{g})=\frac{k_{\mathrm{rl}}(S, \vec{g}) / \mu_{1}(\vec{g})}{k_{\mathrm{rl}}(S, \vec{g}) / \mu_{1}(\vec{g})+k_{\mathrm{rg}}(S, \vec{g}) / \mu_{\mathrm{g}}(\vec{g})}$

306 Initial and boundary conditions for continuous gas 307 injection correspond to given compositions of injected 308 gas and displaced oil:

$C_{i}\left(x_{\mathrm{D}}, 0\right)=C_{i}^{\mathrm{I}}$
$C_{i}\left(0, t_{\mathrm{D}}\right)=C_{i}^{J}$

300 The boundary conditions for the displacement of oil 312 by solvent slug with lean gas drive are:

$C_{i}\left(0, t_{\mathrm{D}}\right): \begin{cases}C_{i}^{\mathrm{J}}, & t_{\mathrm{D}}<1 \\ C_{i}^{\mathrm{D}}, & t_{\mathrm{D}}>1\end{cases}$

313 where $C_{i}^{\mathrm{D}}$ is the composition of gas driving the solvent 315 slug.

316 At this point we introduce new variables:

$\alpha_{i}(\vec{g})=\frac{c_{i 1}-c_{i \mathrm{~g}}}{c_{n 1}-c_{n \mathrm{~g}}}, i=2,3, \ldots n-1$

$\beta_{i}(\vec{g})=c_{i \mathrm{~g}}-\alpha_{i} c_{n \mathrm{~g}}, i=2,3, \ldots n-1$

320 Fig. 1 shows the geometrical meaning of $\alpha_{i}$ and $\beta_{i}$. 321 Vertices $1,2, \ldots, n$ correspond to pure components in 322 phase diagram. Tie line GL connects equilibrium phase 323 compositions, $G_{i} L_{i}$ is the tie line projection on the 324 plane $\left(C_{i}, C_{n}\right)$. The slope of the straight line $G_{i} L_{i}$ is 325 equal to $\alpha_{i}$, the intersection of $G_{i} L_{i}$ with the axes $C_{i}$ is 326 equal to $\beta_{i}$.

327 System (21) takes the form:

$$
\begin{aligned}
& \frac{\partial C}{\partial t_{\mathrm{D}}}+\frac{\partial F(C, \vec{\beta})}{\partial x_{\mathrm{D}}}=0 \\
& \frac{\partial(\vec{\alpha}(\vec{\beta}) C+\vec{\beta})}{\partial t_{\mathrm{D}}}+\frac{\partial(\vec{\alpha}(\vec{\beta}) F+\vec{\beta})}{\partial x_{\mathrm{D}}}=0
\end{aligned}
$$

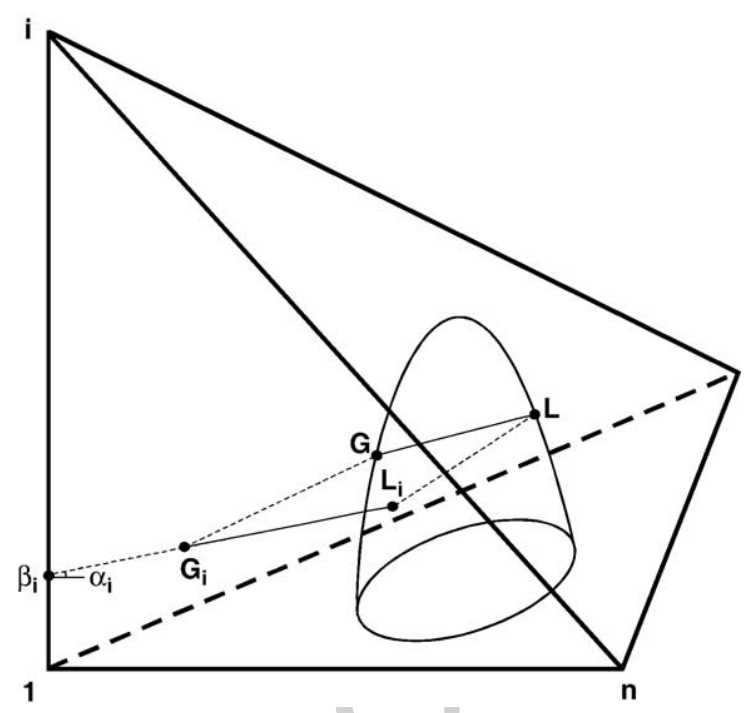

328

Fig. 1. Phase diagram for $n$-component fluids.

In system (29), $C$ is equal to $C_{n}$, the overall volumetric fraction of $n$-th component, and $F$ is equal to $F_{n}$, the overall volumetric fractional flow of $n$-th component.

The unknowns in system (29) of $n-1$ equations are $C$ and $\beta_{i}, i=2,3, \ldots, n-1$.

After the introduction of variables (27) and (28), the initial and boundary conditions (25) for continuous gas injection become

$C\left(x_{\mathrm{D}}, 0\right)=C_{n}^{\mathrm{I}}$

$\beta_{i}\left(x_{\mathrm{D}}, 0\right)=\beta_{i}\left(\vec{g}^{\mathrm{I}}\right)$

$C\left(0, t_{\mathrm{D}}\right)=C_{n}^{\mathrm{J}}$

$\beta_{i}\left(0, t_{\mathrm{D}}\right)=\beta_{i}\left(\vec{g}^{\mathrm{J}}\right)$

For displacement of oil by a rich gas slug with lean gas drive, the boundary conditions (26) take the form

$C\left(0, t_{\mathrm{D}}\right): \begin{cases}C_{n}^{\mathrm{J}}, & t_{\mathrm{D}}<1 \\ C_{n}^{\mathrm{D}}, & t_{\mathrm{D}}>1\end{cases}$

$\beta_{i}\left(0, t_{\mathrm{D}}\right): \begin{cases}\beta_{i}(\vec{g} \mathrm{~J}), & t_{\mathrm{D}}<1 \\ \beta_{i}(\vec{g} \mathrm{D}), & t_{\mathrm{D}}>1\end{cases}$

The conservation law form of the first Eq. (29) allows the introduction of the following potential:

362

$c=-\frac{\partial \varphi}{\partial x_{\mathrm{D}}}, F=\frac{\partial \varphi}{\partial t_{\mathrm{D}}}$

The potential $\varphi\left(x_{\mathrm{D}}, t_{\mathrm{D}}\right)$ is equal to the $n$-th component volume flowing via a trajectory connecting points $(0,0)$ and $\left(x_{\mathrm{D}}, t_{\mathrm{D}}\right)$ : 
372 and the integral (34) is a function of $x_{\mathrm{D}}$ and $t_{\mathrm{D}}$, and is 373 independent of the trajectory.

$374 \quad$ Let us introduce the variable

$\psi=x_{\mathrm{D}}-t_{\mathrm{D}}$

376 From the incompressibility of the total flux follows 378 that $\psi\left(x_{\mathrm{D}}, t_{\mathrm{D}}\right)$ is equal to the overall mixture volume 379 flowing via a trajectory connecting points $(0,0)$ and $380\left(x_{\mathrm{D}}, t_{\mathrm{D}}\right)$.

381 After the following transformation of independent 382 variables

$\Theta:\left(x_{\mathrm{D}}, t_{\mathrm{D}}\right) \rightarrow(\psi, \varphi)$

383 system (29) becomes

$\frac{\partial}{\partial \varphi}\left(\frac{C}{F-C}\right)-\frac{\partial}{\partial \psi}\left(\frac{1}{F-C}\right)=0$

$385 \frac{\partial \vec{\beta}}{\partial \varphi}+\frac{\partial \vec{\alpha}(\vec{\beta})}{\partial \psi}=0$

388 Derivation of system (38) is presented in Appendix 389 B. The most important feature of the system (37), (38) 390 is the independence of the $n-2$ Eq. (38) from the first 391 Eq. (37). The unknowns in the system (38) are $\beta_{i}, i=2$, $3923, \ldots, n-1$. The hyperbolic Eq. (37) contains the un393 known $C(\psi, \varphi)$ and the known vector function $394 \beta_{i}(\psi, \varphi)$, which is the solution of (38).

395 The system (38) is called the auxiliary system of the 396 large system (29). It is important to mention that the 397 system (29) contains thermodynamic functions and 398 transport properties, while the auxiliary system contains 399 only thermodynamic functions.

400 The initial and boundary conditions (30), (31) and 401 (32) allow the calculation of both potentials along the 402 axes $x_{\mathrm{D}}$ and $t_{\mathrm{D}}$ where the conditions are set.

403 Performing the integration (34) in $x_{\mathrm{D}}$ accounting 404 for (30) we obtain the potential $\varphi$ along the axes $405 x_{\mathrm{D}}$ :

$$
t_{\mathrm{D}}=0: \varphi=0-C_{n}^{\mathrm{I}} \psi
$$$$
\psi=x_{\mathrm{D}}
$$

408 So, the initial conditions (30) in coordinates $(\psi, \varphi)$ 409 become

$$
\begin{aligned}
\varphi & =-C^{\mathrm{I}} \psi: C=C^{\mathrm{I}} \\
410 \varphi & =-C^{\mathrm{I}} \psi: \vec{\beta}=\vec{\beta}^{\mathrm{I}}
\end{aligned}
$$

Integrating (34) in $t_{\mathrm{D}}$ accounting for boundary condition (31) allows calculation of the potential $\varphi$ along the axes $t_{\mathrm{D}}$ :

$x_{\mathrm{D}}=0: \varphi=0-F_{n}^{\mathrm{J}} \psi$

$\psi=-t_{\mathrm{D}}$

The boundary conditions (31) take the form:

$\varphi=-F^{\mathrm{J}} \psi: C=C^{\mathrm{J}}$

$\varphi=-F^{\mathrm{J}} \psi: \vec{\beta}=\vec{\beta}^{\mathrm{J}}$

The boundary condition (32) for slug injection gives the following value of potential $\varphi$ :

$x_{\mathrm{D}}=0: \varphi\left\{\begin{array}{l}-F_{n}^{\mathrm{J}} \psi,-1<\psi<0 \\ F_{n}^{\mathrm{J}}-F_{n}^{\mathrm{D}}(\psi+1),-\infty<\psi<-1\end{array}\right.$

So, the boundary conditions (32) become:

428

$C=\left\{\begin{array}{l}C^{\mathrm{J}}, \varphi=-F^{\mathrm{J}} \psi,-1<\psi<0 \\ C^{\mathrm{D}}, \varphi=-F^{\mathrm{J}}-F^{\mathrm{D}}(\psi-1),-\infty<\psi<-1\end{array}\right.$

$\vec{\beta}=\left\{\begin{array}{l}\vec{\beta}^{\mathrm{J}}, \varphi=-F^{\mathrm{J}} \psi,-1<\psi<0 \\ \vec{\beta}^{\mathrm{D}}, \varphi=-F^{\mathrm{J}}-F^{\mathrm{D}}(\psi-1),-\infty<\psi<-1\end{array}\right.$

Therefore, the transformation (36) separates the initial and boundary conditions for the large system (29) into initial-boundary value problem for auxiliary system (38) and the initial-boundary value problem for the lifting Eq. (37).

It is worth mentioning that the elementary wave speeds of the auxiliary system are linked with the wave speeds of the large system by

$D=\frac{F+V}{C+V}$

The eigenvalues of the large and auxiliary systems for $\beta$ waves are related by:

$\Lambda_{k}(C, \vec{\beta})=\frac{F+1 / \lambda_{k}(\vec{\beta})}{C+1 / \lambda_{k}(\vec{\beta})}$,

$k=2,3, \ldots, n-1$.

The phase transitions occurring during gas-based EOR displacements throughout the 1D reservoir are determined just by thermodynamics of the oil-gas system and are independent of transport properties.
430

441 
448 The solution of the large system $\beta_{i}\left(x_{\mathrm{D}}, t_{\mathrm{D}}\right)$ realizes the 449 mapping from the plane $\left(x_{\mathrm{D}}, t_{\mathrm{D}}\right)$ to the set of tie lines in $n$ 450 vertices simplex of $n$-component phase diagram. The 451 image of the domain of the plane $\left(x_{\mathrm{D}}, t_{\mathrm{D}}\right) ; x_{\mathrm{D}}>0$, $452 t_{\mathrm{D}}>0$, defines $2 \mathrm{D}$ surfaces in the simplex. The auxiliary 453 solution $\beta_{i}(\psi, \varphi)$ also maps the domain of the plane $(\psi$, $454 \varphi$ ), where the initial-boundary value problem is defined, 455 into 2D surface in the simplex. From the splitting of the 456 compositional model (29) into auxiliary (38) and lifting 457 (37) problems follow that these surfaces coincide.

458 The auxiliary solution depends on thermodynamic 459 functions $\alpha_{i}$ and $\beta_{i}$ and on the composition fractions of 460 the initial and boundary conditions. So, the $2 \mathrm{D}$ solution 461 image in the simplex is independent of transport prop462 erties, i.e. fractional flow curves, relative phase perme463 ability and phase viscosities.

\section{2.3. Wag injection}

465 During miscible WAG (water-alternate-gas) flooding, 466 aqueous phase contains just water component, and oleic 467 phase is an $n$-component mixture of the virgin oil with 468 hydrocarbon components of the gaseous solvent:

$$
\begin{aligned}
& \frac{\partial s}{\partial t_{\mathrm{D}}}+\frac{\partial f(s, \vec{c})}{\partial x_{\mathrm{D}}}=0 \\
& \frac{\partial(\vec{c} s)}{\partial t_{\mathrm{D}}}+\frac{\partial \vec{c} f(s, \vec{c})}{\partial x_{\mathrm{D}}}=0
\end{aligned}
$$

460 Here $\vec{c}$ is an $n$-vector of hydrocarbon components in 472 the oleic phase and $\mathrm{s}$ is saturation of oleic phase. When 473 gas composition in all slugs is the same, the problem (50) 474 is equivalent to the case of binary oil-gas mixture. 475 System (50) is mathematically equivalent to the 476 system of multi component polymer flooding with no 477 adsorption, $\vec{\alpha}(\vec{c})=0$. So, the introduction of potential 478 (8) transforms the system (50) into the form

$$
\frac{\partial \vec{c}\left(x_{\mathrm{D}}, \varphi\right)}{\partial x_{\mathrm{D}}}=0
$$

480 The proposed splitting technique significantly sim482 plifies exact solution for miscible WAG if compared 483 with that derived in Bedrikovetsky (1993).

\section{2.4. Carbonised waterflooding}

485 Displacement of oil by carbonised water is described 486 by $(n+1) \times(n+1)$ hyperbolic system

$$
\begin{aligned}
& \frac{\partial s}{\partial t_{\mathrm{D}}}+\frac{\partial f(s, \vec{c})}{\partial x_{\mathrm{D}}}=0 \\
& \frac{\partial(\vec{c} s+\vec{b}(\vec{c})(1-s))}{\partial t_{\mathrm{D}}}+\frac{\partial(\vec{c} f(s, \vec{c})+\vec{b}(\vec{c})(1-f))}{\partial x_{\mathrm{D}}}=0
\end{aligned}
$$

Here low concentration of gases in injected water $\vec{c}$ and low equilibrium concentration of gases in oil $\vec{b}$ $(\vec{c})$ do not change overall volume balance of water and oil phases if compared with immiscible waterflooding.

The introduction of coordinates $\varphi$ and $\psi,(8)$ and (35), results in the following $(n) \times(n)$ auxiliary system

$\frac{\partial \vec{b}(\vec{c})}{\partial \varphi}+\frac{\partial(\vec{c}-\vec{b})}{\partial \Psi}=0$.

2.5. Hot waterflood with heat losses for surround formations

Displacement of oil by hot/cold water is described by a $(2) \times(2)$ hyperbolic system of quasi-linear equations of water volume balance and of heat balance for water-oil-rock system

$$
\begin{aligned}
& \frac{\partial s}{\partial t_{\mathrm{D}}}+\frac{\partial f(s, T)}{\partial x_{\mathrm{D}}}=0 \\
& \frac{\partial(T(s+b))}{\partial t_{\mathrm{D}}}+\frac{\partial(T(f+h))}{\partial x_{\mathrm{D}}}=\alpha(T-1)
\end{aligned}
$$

where $T$ is the temperature. A quasi steady state heat flux from the reservoir into surround formations (Newton's law) is assumed, and $\alpha$ is a heat transfer coefficient.

Introduction of potential $\varphi(8)$ and $\psi=b x_{\mathrm{D}}-h t_{\mathrm{D}}$ results in the linear auxiliary equation

$\frac{\partial T}{\partial \varphi}+\frac{\partial T}{\partial \Psi}=-\alpha(T-1)$

and the solution of the auxiliary problem (55) decreases along the characteristic lines $\varphi-\psi=$ constant with decrement $\alpha$. It allows derivation of the exact solution for alternate injection of hot and cold water in oil reservoir accounting for heat losses.

\section{An analytical model for oil displacement by polymer slug with water drive}

503

505

506

507

508

509

510

512

513

514

515

516

517

In this section the splitting technique is applied to the analytical modelling of oil displacement by a polymer slug with water drive. The same procedure may be applied to the solution of the problem of gas slug injection with lean gas drive.

We assume a linear sorption isotherm $a(c)=\Gamma c$. Typical fractional flow functions are shown in Fig. 2.

The chemical flooding problem with only one chemical component in solution is a $(2) \times(2)$ hyperbolic system. For the linear adsorption isotherm con-

89 90 91 92 93 94

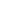

(1)

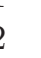




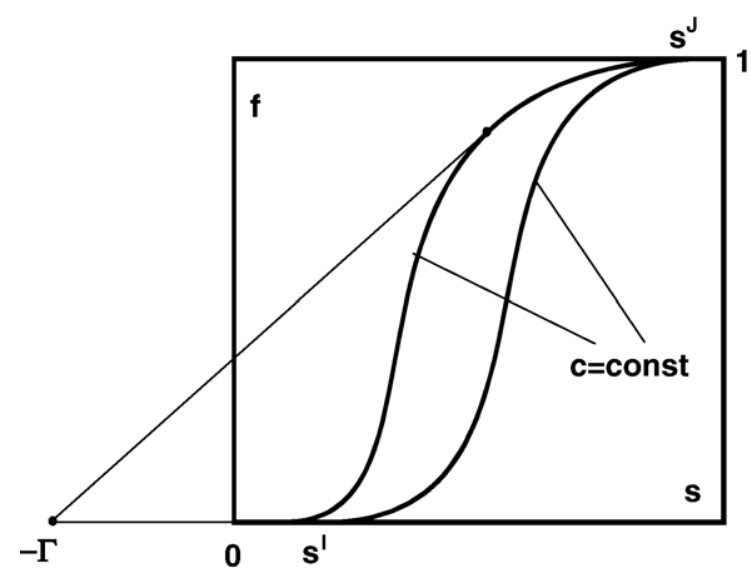

Fig. 2. Typical forms of fractional flow curves.

528 sidered here, the auxiliary system is a linear hyper529 bolic equation:

$\Gamma \frac{\partial c}{\partial \varphi}+\frac{\partial c}{\partial x}=0$

530 subject to the initial and boundary conditions

$$
\begin{aligned}
& \varphi=-s^{\mathrm{I}} x_{\mathrm{D}}: c=0 \\
& x_{\mathrm{D}}=0: c(0, \varphi)=\left\{\begin{array}{l}
1,0<\varphi<1 \\
0,1<\varphi<+\infty
\end{array}\right.
\end{aligned}
$$

532 The solution of the auxiliary problem is given by: $c\left(x_{\mathrm{D}}, \varphi\right)=\left\{\begin{array}{l}0,-s^{\mathrm{I}} x_{\mathrm{D}}<\varphi<\Gamma x_{\mathrm{D}} \\ 1, \Gamma x_{\mathrm{D}}<\varphi<\Gamma x_{\mathrm{D}}+1 \\ 0, \Gamma x_{\mathrm{D}}+1<\varphi<+\infty\end{array}\right.$

536 For the sake of simplicity, we define two new de537 pendent variables for the lifting Eq. (10):

$$
U=\frac{1}{f}, F(U, \vec{c})=-\frac{s}{f}
$$

538 that becomes

$$
\frac{\partial U}{\partial x_{\mathrm{D}}}+\frac{\partial F(U, \vec{c})}{\partial \varphi}=0
$$

540 The lifting problem for these new variables corre543 sponds to the following boundary conditions:

$$
\begin{aligned}
& x_{\mathrm{D}}=0 ; U=1 \\
& \varphi=-s^{\mathrm{I}} x_{\mathrm{D}}: U=+\infty
\end{aligned}
$$

546 There are two discontinuities in the boundary 547 conditions of this problem: at the points $(0,0)$ and $548(0,1)$. The evolution of the discontinuity at the point
$(0,0)$ is given by the path $s^{\mathrm{I}} \rightarrow 3 \rightarrow 2-\left(-s^{\mathrm{J}}\right)$. Fig. 3 shows the solution path through two fractional flow functions $f(s, c)$ in new variables $U$ and $F$. The speed of the shock $s^{\mathrm{I}} \rightarrow 3$ is equal to $\left(-s^{\mathrm{I}}\right)^{-1}$. The speed of the shock $3 \rightarrow 2$ is $1 / \Gamma$, and point 2 is a tangent point of the curve $F=F(U, c=1)$ and the straight line 2-3.

$\frac{U_{2}-U_{3}}{F_{2}-F_{3}}=\frac{1}{F_{U}^{\prime}\left(U_{2}, 1\right)}=\Gamma$

549

550

551

552

553

554

555

The area between the fronts $\varphi=\Gamma x_{\mathrm{D}}$ and $\varphi=\Gamma x_{\mathrm{D}}+1$ is filled by the $s$-wave $2-\left(-s^{\mathrm{J}}\right)$. The values $U^{+}$ ahead of the front $\varphi=\Gamma x_{\mathrm{D}}+1$ are determined by the $s$-wave

$U=U^{0}\left(\frac{\varphi^{\prime}}{x_{\mathrm{D}}^{\prime}}\right), F_{U}^{\prime}\left(U^{0}, c=1\right)=\frac{\varphi^{\prime}}{x^{\prime}{ }_{\mathrm{D}}}$

The points ahead of and behind the shock, $U^{+}$and $U^{-}$, are linked by the Hugoniot-Rankine conditions:

$56 B$

$\Gamma=\frac{F\left(U^{+}, 1\right)-F\left(U^{-}, 0\right)}{U^{+}-U^{-}}$

In the domain behind the shock $\varphi=\Gamma x_{\mathrm{D}}+1$, the values of $U$ are constant along the $s$-characteristics:

$U\left(x_{\mathrm{D}}, \varphi\right)=U^{-}\left(x_{\mathrm{D}}^{\prime}, \varphi^{\prime}\right)$

$\frac{\varphi-\varphi^{\prime}}{x_{\mathrm{D}}-x_{\mathrm{D}}^{\prime}}=F_{U}^{\prime}\left(U^{-}, 0\right)$

Now we consider the $s$-characteristic passing through a point $\left(x_{\mathrm{D}}, \varphi\right)$ from the area behind the shock $\varphi=\Gamma x_{\mathrm{D}}+1$. This characteristic crosses the front $\varphi=\Gamma x_{\mathrm{D}}+1$ at the point $\left(x_{\mathrm{D}}^{\prime}, \varphi^{\prime}\right)$ (Fig. 4). So, the system of four transcendental equations (63) (64) and (65)

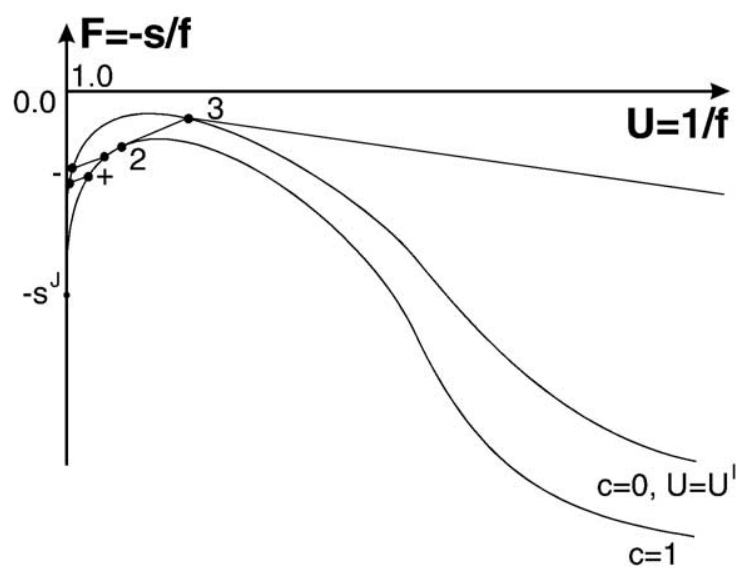

Fig. 3. The lifting problem in plane $(U, F)$. 


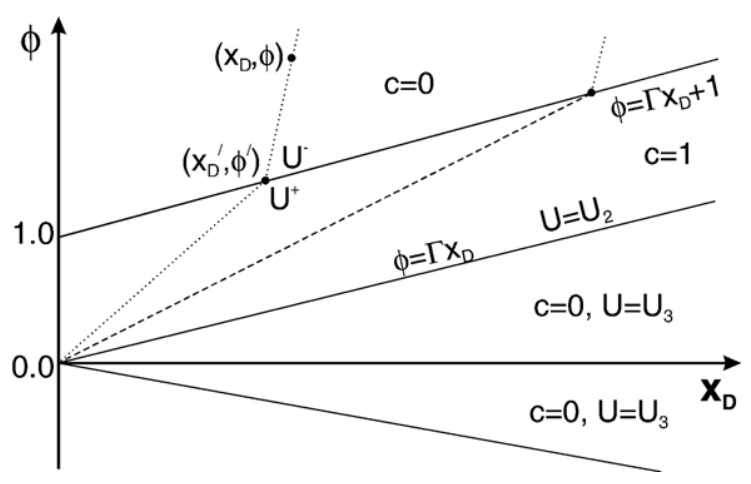

Fig. 4. Solution of the auxiliary and lifting problem.

576 determines the unknowns $x_{\mathrm{D}}^{\prime}, \varphi^{\prime}, U^{-}$and $\mathrm{U}^{+}$for given $577 x_{\mathrm{D}}$ and $\varphi$.

578 The solution of the lifting problem is given by the 579 formula:

$$
U\left(x_{\mathrm{D}}, \varphi\right)= \begin{cases}U_{3} & -s^{\mathrm{I}} x<\varphi<\Gamma x_{\mathrm{D}} \\ U^{0}\left(\frac{\varphi}{x_{\mathrm{D}}}\right) & \Gamma x_{\mathrm{D}}<\varphi<\Gamma x_{\mathrm{D}}+1 \\ U^{-}\left(x_{\mathrm{D}}, \varphi\right) & \Gamma x_{\mathrm{D}}+1<\varphi<+\infty\end{cases}
$$

58@ The expression of unknown $s$ via $U$ is obtained from 583 (59):

$$
s=-U F(U, c)
$$

586 Finally, the solution $s\left(x_{\mathrm{D}}, \varphi\right)$ is:

$$
s\left(x_{\mathrm{D}}, \varphi\right)= \begin{cases}s_{3} & -s^{\mathrm{I}} x_{\mathrm{D}}<\varphi<\Gamma x_{\mathrm{D}} \\ s^{0}\left(\frac{\varphi}{x_{\mathrm{D}}}\right) & \Gamma x_{\mathrm{D}}<\varphi<\Gamma x_{\mathrm{D}}+1 \\ s^{-}\left(x_{\mathrm{D}}, \varphi\right) & \Gamma x_{\mathrm{D}}+1<\varphi<+\infty\end{cases}
$$

588 In order to invert the mapping (9), we calculate the 590 variable $t_{\mathrm{D}}\left(x_{\mathrm{D}}, \varphi\right)$ from (8). In the area ahead of the 591 front $\varphi=\Gamma x_{\mathrm{D}}$, the dependent variables $s$ and $f$ are 592 constant:

$$
t_{\mathrm{D}}=\frac{1}{f_{3}} \int_{0}^{\varphi} d \varphi^{\prime}+\frac{s_{3}}{f_{3}} \int_{0}^{x_{\mathrm{D}}} d x^{\prime}
$$

593 Repeating the integration in the area between fronts $596 \varphi=\Gamma x_{\mathrm{D}}$ and $\varphi=\Gamma x_{\mathrm{D}}+1$, where $s$ and $f$ are constant 597 along each characteristic line, we get:
Next we determine time $t$ along the front $\varphi=\Gamma x_{\mathrm{D}}+1$. The expressions linking $x_{\mathrm{D}}$ and $\varphi$ with the variable $s$ ahead of the front are:

$$
\begin{aligned}
& \varphi=\Gamma x_{0}(\varphi)+1 \\
& \frac{\varphi}{x_{0}(\varphi)}=\frac{f\left(s^{+}, 1\right)-s^{+} f_{s}^{\prime}\left(s^{+}, 1\right)}{f_{s}^{\prime}\left(s^{+}, 1\right)}
\end{aligned}
$$

From (71) follows the expression for $x_{0}(\varphi)$ in a parametric form:

$$
\begin{aligned}
x_{0}\left(s^{+}\right) & =\frac{f^{\prime}\left(s^{+}, 1\right)}{f\left(s^{+}, 1\right)-f^{\prime}\left(s^{+}, 1\right)\left(\Gamma+s^{+}\right)} \\
\varphi\left(s^{+}\right) & =\frac{f^{\prime}\left(s^{+}, 1\right)-s^{+} f^{\prime}\left(s^{+}, 1\right)}{f\left(s^{+}, 1\right)-f^{\prime}\left(s^{+}, 1\right)\left(\Gamma+s^{+}\right)}
\end{aligned}
$$

then, along the front

$$
\begin{aligned}
& t_{\mathrm{D}}=\frac{1}{f\left(s^{+}, 1\right)-f^{\prime}\left(s^{+}, 1\right)\left(\Gamma+s^{+}\right)} \\
& x_{0}\left(t_{\mathrm{D}}\right)=\frac{f^{\prime}\left(s^{+}, 1\right)}{f\left(s^{+}, 1\right)-f^{\prime}\left(s^{+}, 1\right)\left(\Gamma+s^{+}\right)}
\end{aligned}
$$

Fig. 4 shows $s$-characteristics of the lifting equation ahead of and behind the rear front $x_{0}(\varphi)$.

The final expression for $t_{\mathrm{D}}\left(x_{\mathrm{D}}, \varphi\right)$ is:

$$
t_{\mathrm{D}}\left(x_{\mathrm{D}}, \varphi\right)= \begin{cases}\frac{\varphi}{f_{3}}+\frac{s_{3}}{f_{3}} x_{\mathrm{D}} & -s^{\mathrm{I}} x_{\mathrm{D}}<\varphi<\Gamma x_{\mathrm{D}} \\ \frac{\varphi}{f\left(s^{0}\left(\frac{\varphi}{x_{\mathrm{D}}}\right), 1\right)}+\frac{s^{0}\left(\frac{\varphi}{x_{\mathrm{D}}}\right)}{f\left(s^{0}\left(\frac{\varphi}{x_{\mathrm{D}}}\right), 1\right)} x_{\mathrm{D}} & \Gamma x_{\mathrm{D}}<\varphi<\Gamma x_{\mathrm{D}}+1 \\ \frac{\varphi}{f\left(s^{-}\left(x_{\mathrm{D}}, \varphi\right), 0\right)}+\frac{s^{-}\left(x_{\mathrm{D}}, \varphi\right)}{f\left(s^{-}\left(x_{\mathrm{D}}, \varphi\right), 0\right)} x_{\mathrm{D}} & \Gamma x_{\mathrm{D}}+1<\varphi<+\infty\end{cases}
$$

Finally, the solution for $c\left(x_{\mathrm{D}}, t_{\mathrm{D}}\right)$ and $s\left(x_{\mathrm{D}}, t_{\mathrm{D}}\right)$ is given by the following expressions:

$$
c\left(x_{\mathrm{D}}, t_{\mathrm{D}}\right)=\left\{\begin{array}{l}
0, \frac{\left(s_{3}-s^{\mathrm{I}}\right)}{f_{3}} x_{\mathrm{D}}<t_{\mathrm{D}}<\frac{\left(s_{3}+\Gamma\right)}{f_{3}} x_{\mathrm{D}} \\
1, \frac{\left(s^{0}\left(\frac{x_{\mathrm{D}}}{t_{\mathrm{D}}}\right)+\Gamma\right)}{f\left(s^{0}\left(\frac{x_{\mathrm{D}}}{t_{\mathrm{D}}}\right)\right)} x_{\mathrm{D}}<t_{\mathrm{D}}<\frac{\left(s^{0}\left(\frac{x_{\mathrm{D}}}{t_{\mathrm{D}}}\right)+\Gamma\right) x_{\mathrm{D}}+f\left(s^{0}\left(\frac{x_{\mathrm{D}}}{t_{\mathrm{D}}}\right)\right)}{f\left(s^{0}\left(\frac{x_{\mathrm{D}}}{t_{\mathrm{D}}}\right)\right)} \\
0, \frac{\left(s^{-}\left(x_{\mathrm{D}}, t_{\mathrm{D}}\right)+\Gamma\right) x_{\mathrm{D}}+f\left(s^{-}\left(x_{\mathrm{D}}, t_{\mathrm{D}}\right)\right)}{f\left(s^{-}\left(x_{\mathrm{D}}, t_{\mathrm{D}}\right)\right)}<t_{\mathrm{D}}<+\infty
\end{array}\right.
$$

$$
s\left(x_{\mathrm{D}}, t_{\mathrm{D}}\right)= \begin{cases}s_{3} & \frac{\left(s_{3}-s^{\mathrm{I}}\right)}{f_{3}} x_{\mathrm{D}}<t_{\mathrm{D}}<\frac{\left(s_{3}+\Gamma\right)}{f_{3}} x_{\mathrm{D}} \\ s^{0}\left(\frac{x_{\mathrm{D}}}{t_{\mathrm{D}}}\right) & \frac{\left(s^{0}\left(\frac{x_{\mathrm{D}}}{t_{\mathrm{D}}}\right)+\Gamma\right)}{f\left(s^{0}\left(\frac{x_{\mathrm{D}}}{t_{\mathrm{D}}}\right)\right)} x_{\mathrm{D}}<t_{\mathrm{D}}<\frac{\left(s^{0}\left(\frac{x_{\mathrm{D}}}{t_{\mathrm{D}}}\right)+\Gamma\right) x_{\mathrm{D}}+f\left(s^{0}\left(\frac{x_{\mathrm{D}}}{t_{\mathrm{D}}}\right)\right)}{f\left(s^{0}\left(\frac{x_{\mathrm{D}}}{t_{\mathrm{D}}}\right)\right)} \\ s^{-}\left(x_{\mathrm{D}}, t_{\mathrm{D}}\right) & \frac{\left(s^{-}\left(x_{\mathrm{D}}, t_{\mathrm{D}}\right)+\Gamma\right) x_{\mathrm{D}}+f\left(s^{-}\left(x_{\mathrm{D}} t_{\mathrm{D}}\right)\right)}{f\left(s^{-}\left(x_{\mathrm{D}}, t_{\mathrm{D}}\right)\right)}<t_{\mathrm{D}}<+\infty\end{cases}
$$

600

601

602

603

606

808 
621

From now we use the following dimensionless space 622 and time:

$x_{\mathrm{D}}=\frac{\Phi x}{\Delta}, t_{\mathrm{D}}=\frac{u t}{\Delta}$

623 where $\Delta$ is the slug volume.

625 The graphical solution of the problem (5), (6) is 626 presented in Fig. 5. Fig. 6 shows movements of con627 centration and saturation fronts in plane $\left(x_{\mathrm{D}}, t_{\mathrm{D}}\right)$. The 628 shock speeds $D_{2}$ and $D_{3}$ are given by:

$$
\begin{aligned}
& D_{2}=\frac{f_{2}}{s_{2}+\Gamma}=\frac{f_{3}}{s_{3} \Gamma} \\
& D_{3}=\frac{f_{3}}{s_{3}-s^{I}}
\end{aligned}
$$

639 and are obtained graphically in plane $(s, f)$. Here $D_{2}$ is 631 the velocity of the oil bank, $D_{3}$ is the slug front 632 velocity.

633 Trajectory of the rear slug front is given by the 634 parametric formulae (73)). The explicit dependency $635 x_{0}\left(t_{\mathrm{D}}\right)$ can be found geometrically. Draw the tangent 636 to the fractional flow curve $c=1$ at point $s^{+}\left(x_{0}\right)$ to meet 637 axis $f$ at point $\mathrm{A}$ and axis $s$ at point $\mathrm{B}$. Then

$A_{0}=\frac{1}{t_{\mathrm{D}}}, B_{0}=\frac{1}{x_{0}\left(t_{\mathrm{D}}\right)}$

630 Let us fix time $t_{\mathrm{D}}$ and calculate $A_{0}$. From (79) it 641 follows that if the segment $A_{0}$ is marked up and the 642 tangent to the curve $c=1$ is drawn from the point A, 643 then it meets the curve 1 at the point $s^{+}\left(x_{0}\right)$, and the 644 intersection with the axes $s$ at point B defines the 645 coordinate $x_{0}\left(t_{\mathrm{D}}\right)$. The straight line $(-\Gamma)-s^{+}\left(x_{0}\right)$ is

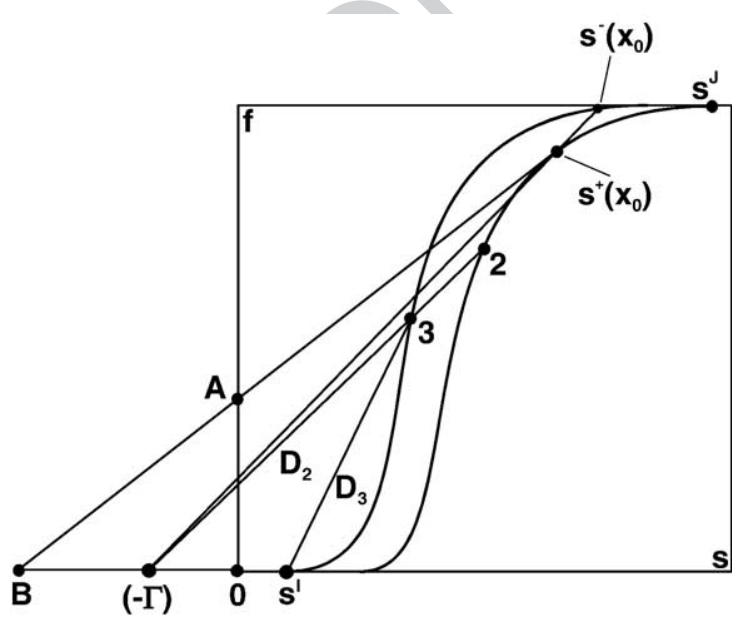

Fig. 5. Solution of the slug problem in the phase plane $(s, f)$.

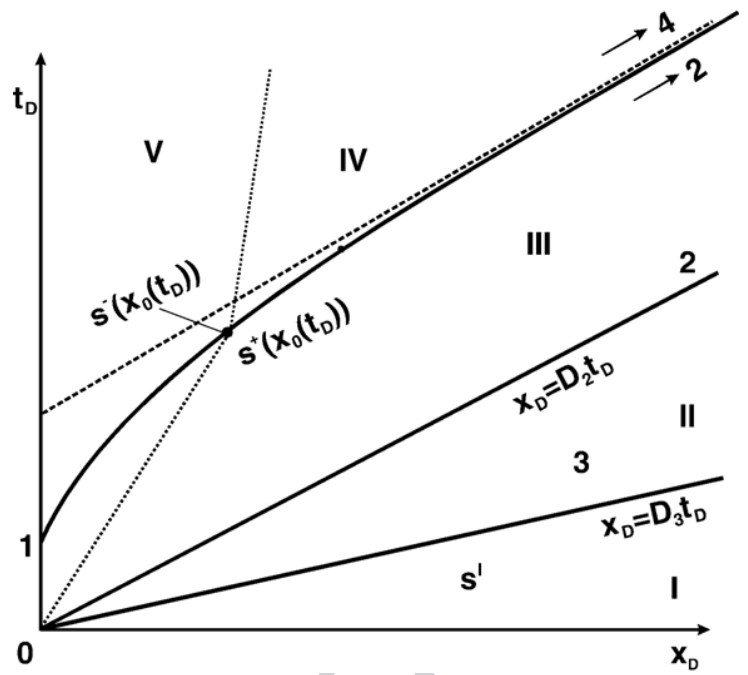

Fig. 6. Trajectories of fronts in plane $\left(x_{\mathrm{D}}, t_{\mathrm{D}}\right)$ for the slug problem.

then produced to meet the curve $c=0$ at point $s^{-}\left(x_{0}\right)$.

The structure of the displacement zone during polymer slug injection (Fig. 6) is:

I. Zone of displaced oil, $c=0, s=s^{\mathrm{I}}$;

II. Water-oil bank formed ahead of the slug, $c=0$, $s=s_{3}$, velocity of the leading front of the bank is $D_{3}$;

664

665

666

667

668

669

670

671

672

III. Polymer slug, $c=1$, saturation decreases from $s^{+}\left(x_{0}\right)$ ahead of the rear front of the slug up to $s_{2}$ on the leading front of the slug; the leading slug front velocity is equal to $D_{2}$;

IV. Water drive zone with mobile oil; $c=0$, saturation decreases from $s^{\mathrm{J}}$ at the stagnant front up to $s^{-}\left(x_{0}\right)$ behind the rear slug front; the position of the stagnant front is determined by equality $s^{-}\left(x_{0}\right)=s^{\mathrm{J}}$;

V. Water drive zone with immobile oil, $s=s^{\mathrm{J}}$.

Sizes of the first, second and fourth zones grow unlimitedly. The slug size grows with time and stabilizes at $t_{\mathrm{D}} \gg 1$. Saturation in slug tends to $s_{3}$; it allows calculating the limit of the slug size from the polymer mass conservation $-1 /\left(s_{3}+\Gamma\right)$. The thickness of the water drive zone with immobile oil becomes constant after the slug rear front passes this zone.

Stabilization of the slug volume with time results in different outcomes, depending on the flow geometry. In case of linear flow (rows of injectors and producers), since the slug volume is proportional to the distance between the leading and rear slug fronts, the slug thickness stabilizes. For radial flow with injection in a single well $x=r^{2} / 2$, and slug
673

674

675

676

677

678

679

680

681

682

683

684

685

686

687

688

689

690

691

692

693

694

695

696

697 
698 thickness tends to zero with order $\left(t_{\mathrm{D}}\right)^{1 / 2}$. This fact 699 should be considered when designing the slug size 700 preventing the slug destruction by more mobile driv701 ing water.

702 Compared with waterflooding, the use of a polymer 703 slug increases the period of water free production, 704 reduces the water cut at initial water drive period, 705 and enhances the ultimate displacement at a stage 706 after breakthrough. Water drive does not disturb the 707 flow ahead of the oil bank and in the front part of the 708 slug.

709 For low sorption (small $\Gamma$ ), the slug injection results 710 in prolongation of water free production while for high 711 sorption slug injection does not change water free 712 period, if compared with waterflooding.

\section{4. Applications}

714 The obtained analytical models for 1D gas injec715 tion and polymer slug flood can be used in stream716 line modelling. The structure of the displacement 717 zone, as obtained from the exact solution, can be 718 used for the interpretation of laboratory and field 719 data.

720 For an $n$-component polymer flooding test, the aux721 iliary system (11) can be used to determine sorption 722 isotherms of each component through relationships 723 linking the sorption isotherms with breakthrough com724 ponent concentrations $c_{i}\left(1, t_{\mathrm{D}}\right)$ measured during the 725 continuous chemical injection. Integrating the left 726 hand side of the auxiliary system over the closed trian727 gle with vortexes in points $(0,0),(1,0)$ and $(1, \varphi)$ with 728 Green's formula

$$
\begin{aligned}
& \int_{\Delta} \int\left(\frac{\partial \vec{a}(\vec{c})}{\partial \varphi}+\frac{\partial \vec{a}}{\partial x_{\mathrm{D}}}\right) \\
& d x_{\mathrm{D}} d \varphi=\int_{\partial \Delta} \vec{c} d \varphi-\vec{a}(\vec{c}) d x_{\mathrm{D}}
\end{aligned}
$$

730 The right hand side integral over the side $(0,0)-(1$, $7320)$ is equal zero due to initial conditions, where all 733 concentrations are zero. The integral over the side $(0$, 734 1)- $(1, \varphi)$ is equal to the mass of the $i$-th component 735 during the production of the volume $\varphi$ of water. The 736 solution of the auxiliary system for continuous polymer 737 injection is self-similar, so $\vec{c}$ is constant along $(0,0)-$ $738(1, \varphi)$. The right hand side of the integral $(80)$ is equal 739 zero:

$$
\int_{0}^{\varphi} \vec{c}(1, y) d y-\vec{c}(1, \varphi) \varphi+\rightarrow a(\vec{c})(1, \varphi)=0
$$

The expression above allows calculating $\rightarrow a(\vec{c})$ for each value of breakthrough concentrations $\vec{c}(1, \varphi)$. The function $\vec{a}(\vec{c})$ is calculated by (81) only along the trajectory $\vec{c}(1, \varphi)$, i.e. sorption isotherms can be determined only for measured concentrations during the test.

Splitting of compositional model into thermodynamics and hydrodynamics equations can be used for testing numerical 1D models. For example, in order to test a polymer simulator, we model two cases that differ from each other by oil viscosity. The timedependencies of accumulated water production $\varphi\left(1, t_{\mathrm{D}}\right)$ and of outlet concentrations $c_{i}\left(1, t_{\mathrm{D}}\right)$ must be different for the two cases, but the outlet concentrations versus accumulated water production $c_{i}(1, \varphi)$ must be the same. The concentrations $c_{i}(1, \varphi)$ must be the same for different oil and water viscosities, relative permeabilities and resistance factors that could vary in wide intervals during the model testing. The concentration equality allows validation of the numerical simulator.

The problem of the compatibility of polymer with formation water can be overcome by the injection of a compatible water slug before the polymer slug injection. In order to avoid contact between the polymer and the formation water, the polymer front should not bypass the compatible waterfront before they both reach the production row $x_{\mathrm{D}}=1$, which could be achieved by the injection of a sufficient volume of compatible water. Determination of the minimum water slug size can be achieved by the solution of the auxiliary system only-if the polymer and compatible water fronts do not meet for $x_{\mathrm{D}}<1$ in the solution of the auxiliary system, they also do not meet in the solution of the general system.

Design of injection gas composition and minimum miscibility pressure calculations may be performed using the auxiliary system only and does not involve transport properties of rock and fluids.

\section{Summary and conclusions}

The $(n+1) \times(n+1)$ system of conservation laws for two-phase $n$-component chemical flooding in porous media with adsorption can be splitted into an $(n) \times(n)$ auxiliary system and one independent lifting equation. The splitting is obtained from the change of independent variables $\left(x_{\mathrm{D}}, t_{\mathrm{D}}\right)$ to $\left(x_{\mathrm{D}}, \varphi\right)$. This change of coordinates also transforms the water conservation law into the lifting equation. In the case of gas/solvent injection, the $(n-1) \times(n-1)$ system of conservation
782

740

743

744

745

746

747

748

749

750

751

752

753

754

755

756

757

758

759

760

761

762

763

764

765

766

767

768

769

770

771

772

773

774

775

776

777

778

779

780

781

783

784

785

786

787

788

789

790

791 
792 laws is splitted into an $(n-2) \times(n-2)$ auxiliary sys793 tem and one independent lifting equation through the 794 change of independent variables $\left(x_{\mathrm{D}}, t_{\mathrm{D}}\right)$ to flow poten795 tials $(\psi, \varphi)$. This change of coordinates transforms the 796 conservation law for the $n$-th component into the lifting 797 equation.

798 The lifting procedure for the solution of the large 799 system consists of:

800

801 - Solution of the auxiliary system;

802 - Solution of the lifting equation;

803 - Inverse transformation of independent variables.

804

805 The auxiliary system contains only equilibrium ther806 modynamic variables, while the large system contains 807 both hydrodynamic (phases relative permeabilities and 808 viscosities) functions and equilibrium thermodynamic 809 variables. Therefore, phase transitions occurring during 810 displacement are determined by the auxiliary system, 811 i.e. they are independent of hydrodynamic properties of 812 fluids and rock. For example, the minimum miscibility 813 pressure (MMP) is independent of relative permeabil814 ities and phase viscosities.

\section{Nomenclature}

$816 a_{i} \quad$ Concentration of $i$-th adsorbed component

$817 b_{i}$ Equilibrium concentration

$818 c_{i}$ Chemical concentration in water, volumetric

819

$820 C$

$821 C_{i}$

$822 D$

$823 f$

$824 F$

825

$826 F_{i}$

827

$828 \vec{g}$

$829 G$

$830 k_{\mathrm{r}}$

$831 l$

$832 L$

$833 n$

$834 s$

$835 S$

$836 t$

$837 T$

$838 t_{\mathrm{D}}$

$839 u$

$840 \mathrm{~V}$

$841 x$

Overall volumetric fraction of $n$-th component Overall volumetric fraction of $i$-th component $x_{0} \quad$ Position of rear slug front

842

$x_{\mathrm{D}} \quad$ Dimensionless distance

843

Greek letters

844

$\alpha \quad$ Geometric parameter of thermodynamic equilibrium

$\beta \quad$ Geometric parameter of thermodynamic equilibrium

$\Delta \quad$ Polymer slug volume, solvent slug volume

$\Phi \quad$ Porosity

$\Gamma \quad$ Proportionality coefficient

$\varphi \quad$ Potential

$\lambda \quad$ Eigenvalue of auxiliary system

$\Lambda \quad$ Eigenvalue of large system

$\mu \quad$ Viscosity

$\Theta \quad$ Transformation of independent variables

$\Omega \quad$ Closed domain

$\psi \quad$ Flow potential of overall flux

845

846

847

848

849

850

851

852

853

854

855

856

857

858

Subscripts

859

g Gas phase

860

i Component index

$\mathrm{k} \quad$ Wave index

1 Liquid phase

Oil phase

Water phase

861

862

863

864

865

Superscripts

866

$+\quad$ Value ahead of the shock

867

- $\quad$ Value behind the shock

D Drive condition

868

869

870

871

$\mathrm{J} \quad$ Injection condition

872

873

$\mathrm{R} \quad$ Inside the slug

874

\section{Acknowledgements}

Authors are grateful to Dr. A. Mailibaev (Institute of Mechanics, Moscow Lomonosov State University) for clearing the physical meaning of the potential and auxiliary system, to Prof. Y. Yortsos (Southern California University) and Prof. D. Marchesin (Institute of Pure and Applied Mathematics, Brazil) for fruitful discussions.

A. P. Pires also thanks the Brazilian Petroleum National Agency (ANP) for partial sponsorship of his Ph.D. research.

Authors thank Eneida Pires, Themis Carageorgos and Nina Gade for support and encouragement.
876 
888

\section{Appendix A. Proof of splitting for chemical flooding}

889 If $s\left(x_{\mathrm{D}}, t_{\mathrm{D}}\right), c_{i}\left(x_{\mathrm{D}}, t_{\mathrm{D}}\right), i=1,2, \ldots, n$ is a solution of 890 system (2), and $\varphi\left(x_{\mathrm{D}}, t_{\mathrm{D}}\right)$ is the potential function (8), 891 then the function $c_{i}\left(x_{\mathrm{D}}, \varphi\right)$ obeys the following conser892 vation law:

$$
\oint_{\partial \Omega} c_{i} d \varphi-a_{i} d x_{\mathrm{D}}=0
$$

893 where $\Omega$ is a closed domain $\Omega \subset R^{2}$.

895 System (2) can be derived from the following con896 servation laws in the integral form:

$$
\oint_{\partial \Omega}\left(c_{i} f\right) d t_{\mathrm{D}}-\left(c_{i} s+a_{i}\right) d x_{\mathrm{D}}=0
$$

898

Applying the definition of the potential (8) in (A-2):

$$
\oint_{\partial \Omega} c_{i}\left(f d t_{\mathrm{D}}-s d x_{\mathrm{D}}\right)-a_{i} d x_{\mathrm{D}}=\oint_{\partial \Omega} c_{i} d \varphi-a_{i} d x_{\mathrm{D}}=0
$$

901 In domains $\Omega$ where the solution is a smooth func903 tion, from the integral conservation laws (A-3) follows 904 the system of partial differential equations (11). In 905 narrow domains around shock trajectories, from (A-3) 906 follows the Hugoniot-Rankine conditions.

\section{Appendix B. Proof of splitting for gas flooding}

908 If $C\left(x_{\mathrm{D}}, t_{\mathrm{D}}\right), \beta_{i}\left(x_{\mathrm{D}}, t_{\mathrm{D}}\right), i=2,3, \ldots, n-1$ is a solu909 tion of system $(26)$, and $\varphi\left(x_{\mathrm{D}}, t_{\mathrm{D}}\right)$ and $\psi\left(x_{\mathrm{D}}, t_{\mathrm{D}}\right)$ are the 910 potential functions (34) and (35), then the function $911 \beta_{i}(\psi, \varphi)$ obeys the following conservation law:

$\oint_{v \partial \Omega} \alpha_{i} d \varphi-\beta_{i} d \psi=0$

912 where $\Omega$ is a closed domain $\Omega \subset R^{2}$.

914 The system (29) was derived from the conservation 915 law of $i$-th component volume balance in the integral 916 form:

$$
\oint_{\partial \Omega}\left(\alpha_{i} F+\beta_{i}\right) d t_{\mathrm{D}}-\left(\alpha_{i} C+\beta_{i}\right) d x_{\mathrm{D}}=0
$$

918 From (B-2), and using the definition of potentials 920 (34) and (35), we obtain:

$$
\begin{gathered}
\oint_{\partial \Omega} \alpha_{i}\left(F d t_{\mathrm{D}}-C d x_{\mathrm{D}}\right)-\beta_{i}\left(d x_{\mathrm{D}}-d t_{\mathrm{D}}\right) \\
\quad=\oint_{\partial \Omega} \alpha_{i} d \varphi-\beta_{i} d \psi=0
\end{gathered}
$$

92B In domains $\Omega$ where the solution is a smooth func924 tion, from the integral conservation law (B-3) follows the system of partial differential equations (38). In narrow domains around shock trajectories, from (B-3) follows the Hugoniot-Rankine conditions.

\section{References}

Barenblatt, G.I., Entov, V.M., Ryzhik, V.M., 1991. Theory of Fluid Flows Through Natural Rocks. Kluwer Academic Publishers, London.

Bedrikovetsky, P.G., 1993. Mathematical Theory of Oil and Gas Recovery. Kluwer Academic Publishers, London.

Bedrikovetsky, P.G., Chumak, M.L., 1992a. Exact solutions for twophase multicomponent displacement. Reports of USSR Academy of Sciences, Series Fluid Mechanics (Transl. From Russian, DAN SSSR, Hydrodynamica) vol. 322, p. 668.

Bedrikovetsky, P.G., Chumak, M.L., 1992b. Riemann problem for two-phase four and more component displacement (ideal mixtures). Proceedings of the Third European Conference on the Mathematics of Oil Recovery (ECMOR), Delft.

Braginskaya, G.S., Entov, V.M., 1980. Non-isothermal displacement of oil by a solution of an active additive. Fluid Dyn. 6, $873-880$

Claridge, E.L., Bondor, P.L., 1974. A graphical method for calculating linear displacement with mass transfer and continuously changing mobilities. Soc. Pet. Eng. J. 14, 609-618.

Dafermos, C.M., 2000. Hyperbolic Conservation Laws in Continuum Physics. Springer Verlag, Berlin.

Dahl, O., Johansen, T., Tveito, A., Winther, R., 1992. Multicomponent Chromatography in a 2-Phase Environment. SIAM J. Appl. Math. 52 (1), 65-104.

de Nevers, N.H., 1964. A calculation method for carbonised waterflooding. Soc. Pet. Eng. J. 4, 9-20.

Dumore, J.M., Hagoort, J., Risseeuw, A.S., 1984. An analytical model for one-dimensional, three-component condensing and vaporizing gas drive. Soc. Pet. Eng. J. 24, 169-179.

Entov, V.M., Voskov, D.V., 2000. On oil displacement by gas injection. Proceedings of the Seventh European Conference on the Mathematics of Oil Recovery (ECMOR), Baveno.

Entov, V.M., Turetskaya, F.D., Voskov, D.V., 2002. On approximation of phase equilibria of multicomponent hydrocarbon mixtures and prediction of oil displacement by gas injection. Proceedings of the Eighth European Conference on the Mathematics of Oil Recovery (ECMOR), Freiberg.

Fayers, J., 1962. Some theoretical results concerning the displacement of a viscous oil by a hot fluid in a porous medium. J. Fluid Mech. $13,65-76$.

Gelfand, I.M., 1959. Some problems in the theory of quasi linear equations (in Russian). Usp. Mat. Nauk 24 (2), 87-158.

Helfferich, F., 1980. Theory of multicomponent multiphase displacement in porous media. Soc. Pet. Eng. J. 21, 51-62.

Hirasaki, G.J., 1981. Application of the theory of multicomponent, multiphase displacement to three-component, two-phase surfactant flooding. Soc. Pet. Eng. J. 21, 191-204.

Johansen, T., Winther, R., 1989. The Riemann problem for multicomponent polymer flooding. SIAM J. Math. Anal. 20 (4), 908-929.

Johansen, T., Tveito, A., Winther, R., 1989. A Riemann solver for a two-phase multicomponent process. SIAM J. Sci. Statist. Comput. $10(5), 846-879$.

Johns, R.T., Orr Jr., F.M., 1996. Miscible gas displacement of multicomponent oils. Soc. Pet. Eng. J. 1 (1), 39-50. 
Orr Jr., F.M., Dindoruk, B., Johns, R.T., 1995. Theory of multicomponent gas/oil displacement. Ind. Eng. Chem. Res. 34, $2661-2669$.

Wachman, C., 1964. A mathematical theory of the displacement of oil and water by alcohol. Soc. Pet. Eng. J. 4, 250-266.

Wang, Y., Orr Jr., F.M., 1997. Analytical calculation of minimum miscibility pressure. Fluid Phase Equilib. 139, 101-124.

Zick, A.A., 1986. A combined condensing/vaporizing mechanism in the displacement of oil by enriched gas. SPE Paper No 15493, Proceedings of the 1986 SPE Annual Technical Conference and Exhibition, New Orleans.

Logan, J.D., 1994. An Introduction to Nonlinear Partial Differential Equations. John Wiley \& Sons, Inc., New York. 